\title{
Probabilistic model of Wind Power Ramp Events Based On Moving Ratio Analysis
}

\author{
Sanming Liu ${ }^{1,}$, Zhijie Wang ${ }^{2, b}$ \\ ${ }^{1}$ Department of Mathematics and Physics , Shanghai Dianji University,Shanghai, 201306, China; \\ ${ }^{2}$ Department of Electrical Engineering, Shanghai Dianji University,Shanghai, 201306, China; \\ a liusanmingxyx@163.com, ${ }^{\mathrm{b}}$ wzjsdstu@163.com
}

\begin{abstract}
Keywords: wind power ramp; three-parameter Weibull distribution; ramp distribution; probabilistic analysis.

Abstract. Description of probability distribution characteristics of wind power ramp events is always a challenge in grid-connected wind power operation analysis. In this paper, a moving ratio based approach of wind power ramp events is introduced and an probability model is described for the wind ramp events. The proposed model is useful to estimate the relationship between power ramp events and the variability of the wind power. The proposed model is able to satisfactorily approximate the actual distribution of wind power ramp events . It is shown that the linear difference based ramp distribution can be calculated using the proposed moving ratio based approach. The proposed model can be an effective tool in planning for wind power ramp control.
\end{abstract}

\section{Introduction}

Sustainable energy resources, especially wind power, are currently increasing in power systems. Advantages of this resource can be summarized as follows: i. Reducing dependence on fossil resources. ii. Reducing greenhouse gases emission. iii.Reducing the energy production cost[1].

In recent years, with the large-scale expansion of wind farms and turbine technology, the percentage of energy obtained from wind sources relative to the peak load is rapidly increasing. As increasing penetration of wind power, wind power brought great threaten to the system stability because of fluctuation and uncertainty of the wind. Especially, the great change of wind power in short time which is called as a ramp is very harmful to the power grid[2-7]. In order to get a reasonable and efficient prediction model to forecast ramp events, each country has done a lot of research.

At present, many scholars should take three main characteristics into account to define a ramp event: direction, duration, and magnitude. According to the three characteristic, different scholars comes up with different definition, four kinds of which are generally accepted by most scholars [7]. Based on the definition of ramp events, R Sevlian and R Rajagopal proposed an effective method for the detection of ramp events in [8], which can detect the possible existence of ramp events by wind power data. On the basis of the detection of ramp events, N Cutler, M Kay, K Jacka, et al used the K-means method to classify the ramp events[9], and H Zareipour, D L Huang, W Rosehart used support vector machine model (SVM) to train the classifier in [10].

Taking into account that the ramp events are described as the wind power changes in a period of time, if the direct use of power data as a sample, the input variable dimension is large. In order to reduce the complexity of matrix computation in the classification process, a number of effective features are proposed to characterize the samples in order to better classify the matrix in [11]. And the change of the amplitude, duration and rate of the climbing definition can be effectively divided into the ramp and non ramp events.

The probabilistic modeling of the wind ramp events plays an important role to analyze the wind power variability and to optimize the fill-in energy requirements. The wind ramp events are generally analyzed as the change (linear difference or ratio ) in generated wind power from minute to minute [11-16]. In this paper, a moving ratio based approach is considered to model wind ramp events. The proposed model generalizes the distribution of ramp events and can be used to estimate the moving 
percentage change in wind power output. Our focus will be abstract and mathematical rather than concrete and empirical.

After this introduction, the rest of the article is structured as follows: In Section 2, the three parameter Weibull distribution model of wind power is derived. In Section 3, the moving ratio based ramp events of wind power variability is described and the analytical model of the ramp events is derived, and the proposed model is discussed. The conclusion is presented in Section 4.

\section{The three parameter Weibull distribution model of wind power}

The probability distribution of wind speed becomes critically important to derive the analytic representation of wind power distribution and to estimate energy production.

There are various statistical distribution functions for describing and analyzing wind data. Some of them include normal, lognormal, Rayleigh and Weibull probability distributions [17,18]. It has been found that among probability distribution functions used by many researchers, the Weibull distribution is most accurate and adequate in wind analyzing and interpreting the actual wind speed data and in predicting the characteristics of prevailing wind profile $[17,19]$.

Two paramete Weibull probability distribution function of wind speed can be expressed mathematically as [20]:

$$
f(v)=\frac{b}{a}\left(\frac{v}{a}\right)^{b-1} \exp \left\{-\left(\frac{v}{a}\right)^{b}\right\},
$$

where $b$ is the Weibull shape factor, $a$ is the scale factor and $v$ is the wind speed under consideration. Weibull distribution can describe distribution of wind speed due to its simple form and easy to calculate. At present, Weibull distribution is used widely in the engineering. The cumulative distribution function $F_{V}(v)$ of (1) can be represented as:

$$
F_{V}(v)=\int_{0}^{v} \frac{b}{a}\left(\frac{x}{a}\right)^{b-1} \exp \left\{-\left(\frac{x}{a}\right)^{b}\right\} d x=1-\exp \left\{-\left(\frac{v}{a}\right)^{b}\right\}
$$

$b$ reflects the peak of the distribution curve. If the variance of the wind speed data is small, then the $b$ value is very high, and the shape of the distribution curve is relatively steep. If $0<b<1$, distribution density is a decreasing function of wind speed. If $b=1$, the distribution function is an exponential function. If $b=2$, the Weibull distribution is called as the Rayleigh distribution. If $b=3.5$, the Weibull distribution is actually close to the normal distribution.

Let $w_{v}$ represents the power output at the speed $v$, then we have the following[21]

$$
w_{v}= \begin{cases}0 ; & v<v_{I} \\ c v^{n}+d ; & v_{I} \leq v<v_{R} \\ w_{O} ; & v_{R} \leq v \leq v_{O} \\ 0 ; & v_{O}<v\end{cases}
$$

where $v_{I}, v_{R}$ and $v_{O}$ are the cut-in wind speed of the turbine, the rated wind speed of the turbine and the cut-out wind speed of the turbine, $c, d, w_{O}, n$ are constants and $n$ is the velocity-power proportionality.

Let $F_{W}(w)$ denote a cumulative distribution which represents the fraction of time that the wind power output of the turbine is equal or lower than a certain value $w\left(w \leq w_{O}\right)$, i.e., 


$$
F_{W}(w)=\operatorname{prob}\left\{w_{v} \leq w \leq w_{O}\right\}
$$

It is obvious that $F_{W}(w)=1$ for $w>w_{O}$. Replacing $w_{v}$ in (4) with $c v^{n}+d$ by (3), we have that

$$
F_{W}(w)=\operatorname{prob}\left\{w_{v} \leq w\right\}=\operatorname{prob}\left\{v \leq\left(\frac{w-d}{c}\right)^{\frac{1}{n}}\right\}
$$

From (2), we get that

$$
\begin{aligned}
F_{W}(w) & =\operatorname{prob}\left\{w_{v} \leq w\right\}=F_{v}\left(\left(\frac{w-d}{c}\right)^{\frac{1}{n}}\right) \\
& =1-\exp \left\{-\left(\frac{\left(\frac{w-d}{c}\right)^{\frac{1}{n}}}{a}\right)^{b}\right\} \\
& =1-\exp \left\{-\left(\frac{w-d}{a^{n} c}\right)^{\frac{b}{n}}\right\},
\end{aligned}
$$

i.e,

$$
F_{W}(w)=1-\exp \left\{-\left(\frac{w-d}{a^{n} c}\right)^{\frac{b}{n}}\right\}
$$

Denoting $\lambda=a^{n} c, \quad k=\frac{b}{n}$, then the cumulative distribution $F_{W}(w)$ defined in (6) can be represented as,

$$
F_{W}(w)=1-\exp \left\{-\left(\frac{w-d}{\lambda}\right)^{k}\right\} .
$$

Therefore the probability density function $f_{W}(w)$ of the cumulative distribution $F_{W}(w)$ can be represented as,

$$
f_{W}(w)=\frac{k}{\lambda}\left(\frac{w-d}{\lambda}\right)^{k-1} \exp \left\{-\left(\frac{w-d}{\lambda}\right)^{k}\right\}
$$

The Eq.(8) whiich represents the probability distribution of the wind power is the three parameter Weibull distribution. 


\section{Distribution of ramp events}

At present, many scholars should take three main characteristics into account to define a ramp event: direction, duration, and magnitude. According to the three characteristic, different people comes up with different definition, four kinds of which are generally accepted by most scholars [7].

With respect to direction, there are two basic types of ramps: upward (or ramp-ups) and downward (or ramp-downs). Upward ramps are characterized by an increase of wind power. The downward ramps result when there is a reduction in wind power. [11-15]. In this paper, the moving ratio based analysis of ramp events is useful to generalize the ramp events.

Let $W(t)$ be the wind power generated at time $t$. It is obvious that the function $W(t)$ is a continuous function of time $t$. Because of feasibility of data acquisition, we can express $W(t)$ as a discrete time event using the fixed time interval. We suppose that the discrete data set of wind power can be represented as

$$
\Lambda=\{W(t) \mid t=0,1,2, \mathrm{~L}, m\},
$$

where $m$ is the total time period under consideration. Let stochastic variable $W(t)$ obey the three parameter Weibull distribution (10). We define a new stochastic variable $q_{\Delta t}$ as

$$
q_{\Delta t}=\frac{W(t+\Delta t)-d}{W(t)-d}, \quad t=0,1,2, \mathrm{~L}, m,
$$

where $\Delta t>0, W(t+\Delta t)$ is the wind power generation at a time $\Delta t$ ahead of $t$. If $q_{\Delta t}>1$, we get an upward ramp. If $q_{\Delta t}<1$, we get an downward ramp. It is obvious that the probability distribution of $q_{\Delta t}$ is the probability distribution of the ramp events.

In the following, we shall derive the probability distribution of $q_{\Delta t}$. Let the cumulative probability distribution function of $q_{\Delta t}$ as $F_{q_{\Delta t}}(r)$. We have that

$$
F_{q_{\Delta t}}(r)=\operatorname{prob}\left(q_{\Delta t} \leq r\right)=\operatorname{prob}\left(\frac{W(t+\Delta t)-d}{W(t)-d} \leq r\right) .
$$

If $W(t)+d=w, \frac{W(t+\Delta t)-d}{W(t)-d} \leq r$ implies $W(t+\Delta t)-d \leq r w$. Therefore we get that

$$
\begin{aligned}
F_{q_{\Delta t}}(r) & =\int_{0}^{+\infty} \operatorname{prob}\{W(t+\Delta t)-d \leq r w\} \cdot \operatorname{prob}\{W(t)-d=w\} d w \\
= & \int_{0}^{+\infty} F_{W}(r w+d) f_{W}(w+d) d w \\
= & \int_{0}^{+\infty}\left[1-\exp \left\{-\left(\frac{r w}{\lambda}\right)^{k(\Delta t)}\right\}\left[\frac{k(\Delta t)}{\lambda}\left(\frac{w}{\lambda}\right)^{k(\Delta t)-1} \exp \left\{-\left(\frac{w}{\lambda}\right)^{k(\Delta t)}\right\} d w\right.\right. \\
= & 1-\int_{0}^{+\infty} \frac{k(\Delta t)}{\lambda}\left(\frac{w}{\lambda}\right)^{k(\Delta t)-1} \exp \left\{-\left(\frac{w}{\lambda}\right)^{k(\Delta t)}\right\} \exp \left\{-\left(\frac{r w}{\lambda}\right)^{k(\Delta t)}\right\} d w \\
& =1-\int_{0}^{+\infty} \frac{k(\Delta t)}{\lambda}\left(\frac{w}{\lambda}\right)^{k(\Delta t)-1} \exp \left\{-\left(1+r^{k}\right)\left(\frac{w}{\lambda}\right)^{k(\Delta t)}\right\} d w
\end{aligned}
$$




$$
\begin{aligned}
& =1+\left.\frac{1}{1+r^{k(\Delta t)}} \exp \left\{-\left(1+r^{k(\Delta t)}\right)\left(\frac{w}{\lambda}\right)^{k(\Delta t)}\right\}\right|_{0} ^{+\infty} \\
& =1-\frac{1}{1+r^{k(\Delta t)}}=\frac{r^{k(\Delta t)}}{1+r^{k(\Delta t)}},
\end{aligned}
$$

i.e., the cumulative probability distribution function of $q_{\Delta t}$ is

$$
F_{q_{\Delta t}}(r)=\frac{r^{k(\Delta t)}}{1+r^{k(\Delta t)}}
$$

and the probability density function of $q_{\Delta t}$ is

$$
f_{q_{\Delta t}}(r)=\frac{k(\Delta t) r^{k(\Delta t)-1}}{\left(1+r^{k(\Delta t)}\right)^{2}}
$$

In (10) and (11), the model parameter $k(\Delta t)$ depends on the time step value $\Delta t$. For different $\Delta t$, the model parameter value changes. The moving ratio based approach of the ramp events is useful to manipulate the distribution of $q_{\Delta t}$ for different time step $\Delta t$.

In the following, we calculate the distribution of the percentage change in moving wind power output using (10) :

$$
\begin{aligned}
& \operatorname{prob}\left\{\left|\frac{W(t+\Delta t)-W(t) \mid}{W(t)-d}\right| \leq(r-1)\right\} \\
& =F_{q_{\Delta t}}(r)-F_{q_{\Delta t}}(2-r) \\
& =\operatorname{prob}\{|W(t+\Delta t)-W(t)| \leq(r-1)(W(t)-d)\} \\
& =\operatorname{prob}\{-(r-1)(W(t)-d) \leq(W(t+\Delta t)-d)-(W(t)-d) \leq(r-1)(W(t)-d)\} \\
& =\operatorname{prob}\{-(r-2)(W(t)-d) \leq(W(t+\Delta t)-d) \leq r(W(t)-d)\} \\
& =\operatorname{prob}\left\{2-r \leq \frac{W(t+\Delta t)-d}{W(t)-d} \leq r\right\}
\end{aligned}
$$

i.e,

$$
\operatorname{prob}\left\{\left|\frac{W(t+\Delta t)-W(t)}{W(t)-d}\right| \leq(r-1)\right\}==\operatorname{prob}\left\{2-r \leq \frac{W(t+\Delta t)-d}{W(t)-d} \leq r\right\} .
$$


Furthermore, the proposed moving ratio based model of the ramp distribution can be used to measure the linear difference based ramp distribution. Let consider a function $G_{\Delta t}(\omega)$ as

$$
G_{\Delta t}(\omega)=\operatorname{Pr} o b\{W(t+\Delta t)-W(t)<\omega\}
$$

Though the linear difference based wind ramp distribution defined in (13) can be represented using Laplace or Gaussian probability distribution, the theoretical description of two parameter analytical model is available in [13]. Using two parameter model, the distribution function of the $G_{\Delta t}(\omega)$ can be represented as [13],

$$
G_{\Delta t}(\omega)=\frac{1}{1+A \exp (-B \omega)}
$$

Here $A$ and $B$ are two model parameters. The moving ratio based approach can be used to generate the ramp distribution as:

$$
\begin{aligned}
& G_{\Delta t}(\omega)=\operatorname{Pr} o b\{W(t+\Delta t)-W(t)<\omega\} \\
& =\operatorname{Pr} o b\{W(t+\Delta t)<\omega+W(t)\} \\
& =\operatorname{Pr} o b\{W(t+\Delta t)-d<\omega+W(t)-d\} \\
& =\int_{W_{\min -d}}^{W_{\max }-d} \operatorname{Pr} o b\left\{\frac{W(t+\Delta t)-d}{W(t)-d}<1+\frac{\omega}{x}\right\} \operatorname{Pr} o b\{W(t)-d=x\} d x
\end{aligned}
$$

i.e,

$$
G_{\Delta t}(\omega)=\int_{W_{\min -d}}^{W_{\max }-d} \operatorname{Pr} o b\left\{\frac{W(t+\Delta t)-d}{W(t)-d}<1+\frac{\omega}{x}\right\} \operatorname{Pr} o b\{W(t)-d=x\} d x .
$$

Using (8) and (9), (14) transforms into

$$
G_{\Delta t}(\omega)=\int_{W_{\min -d}}^{W_{\max }-d} F_{q_{\Delta t}}\left(1+\frac{\omega}{x}\right) f_{W}(d+x) d x
$$

Here $W_{\min }$ and $W_{\max }$ are the lower and upper limit of the distribution under consideration. Using the proposed moving ratio based distribution $F_{q_{\Delta t}}\left(1+\frac{\omega}{x}\right)$, the linear difference based ramp distribution can be numerically calculated using (15). Using a proper choice of the $W_{\min }$ and $W_{\max }$, the proposed moving ratio based distribution is also useful to calculate the linear difference based ramp distribution when the wind power is inside a fixed boundary limit.

\section{Conclusion}

In this paper, a moving ratio based approach is described to analyze the wind power ramp events. An approximate analytical model of the wind ramp events is proposed. The proposed model approxima- 
tes the actual wind power ramp events and is useful to estimate the moving percentage change of wind power output. Taking advantage of the inherent stochastic behavior of wind power output, the analytical model reflects the statistical nature of wind power based on the probability distribution of wind ramp events. Furthermore. It is shown that the linear difference based ramp distribution can be calculated using the proposed moving ratio based approach. The proposed model can be useful in designing power rating, energy capacity and operational policies for utility scale wind power systems.

The moving ratio based approach in the proposed model generalizes the distribution of wind power ramp events and opens a scope to compare the variabilityof different wind power plants based on their ramp distribution.

\section{Acknowledgements}

This work was financially supported by the Innovation Program of Shanghai Municipal Education Commission (No.15ZZ106, No.14YZ157), Shanghai Natural Science Foundation (No. 12ZR1411600, No.14ZR1417200, No.15ZR1417300), Climbing Peak Discipline Project of Shanghai Dianji University(No. 15DFXK01).

\section{References}

[1] C.D. Yue, C.M. Liu and E.M.L. Liou: A transition toward a sustainable energy future feasibility assessment and development strategies of wind power inTaiwan, Energy Policy, Vol. 29 (2001) No. 12, p. 951-963.

[2] N. Francis: Predicting sudden changes in wind power generation, North American Wind Power, Vol. 5 (2008) No.9, p. 58-60.

[3] H. Holttinen, P. Meibom, A. Orths, et al: Impacts of large amounts of wind power on design and operation of power systems, results of IEA collaboration, Wind Energy, Vol. 14 (2011) No. 2, p. 179-192.

[4] T. Aigner, S. Jaehnert, G.L. Doorman, T. Gjengedal: The effect of large-scale wind power on system balancing in Northern Europe, IEEE Transactions on Sustainable Energy, Vol. 3 (2012) No. 4, p. 751-759.

[5] H. Banakar, C. Luo, B.T. Ooi: Impacts of wind power minute-to-minute variations on power system operation, IEEE Transactions on Power Systems, Vol. 23(2008) No. 1, p. 150-160.

[6] N. Navid: Market solutions for managing ramp flexibility with high penetration of renewable resource. IEEE Transactions on Sustainable Energy, Vol. 3 (2012) No. 4, p. 784-790.

[7] C. Ferreira, J. Gama, L. Matias, A. Botterud, J. Wang: A survey on wind power ramp forecasting , Office of Scientific \& Technical Information Technical Reports, Illinois: Argonne National Laboratory(ANL) (2011).

[8] R. Sevlian, R. Rajagopal: Detection and statistics of wind power ramps, IEEE Transactions on Power Systems, Vol. 28 (2013) No. 4, p. 3610-3620.

[9] N. Cutler, M. Kay, K. Jacka, T.S. Nielsen : Detecting, categorizing and forecasting large ramps in wind farm power output using meteorological observations and WPPT, Wind Energy, Vol. 10 (2007) No. 5, p. 453-470.

[10] H. Zareipour, D. Huang, W. Rosehart: Wind power ramp events classification and forecasting : A data mining approach, IEEE Power and Energy Society General Meeting, Vol. 5 (2011) No. 22, p. 1-3. 
[11] C. Gallego, A. Costa, A. Cuerva, L. Landberg, B. Greaves, J. Collins: A wavelet-based approach for large wind power ramp characterization, Wind Energy, Vol. 16 (2013) No. 2, p. 257-278.

[12] C. Kamath: Understanding wind ramp events through analysis of historical data, Transmission and Distribution Conference and Exposition, 2010 IEEE PES in United States: New Orleans, LA (2010).

[13] A.K. Das, B.M. Mazumdar: Statistical model for wind power based on ramp analysis, International Journal of Green Energy (2013).

[14] A. Bossavy, R. Girard, G. Kariniotakis: Forecasting ramps of wind power production with numerical weather prediction ensembles, Wind Energy, Vol. 16 (2013) No. 1, p. 51-63.

[15] B. Kirby, M. Milligan: An exemption of capacity and ramping impacts of wind energy on power systems, Elect J , Vol. 21 (2008) No. 7, p. 30-42.

[16] A.K. Das: An analytical model for ratio based analysis of wind power ramp events, Sustainable Energy Technologies and Assessments. Vol. 9 (2015), p. 49-54.

[17] R.O. Fagbenle, J. Katende, O.O. Ajayi, J.O. Okeniyi: Assessment of wind energy potential of two sites in North-East, Nigeria. Renewable Energy, Vol. 36 (2011) No. 4, p. 1277-1283.

[18] B. Ozerdem, M. Turkeli: An investigation of wind characteristics on the campus of Izmir Institute of Technology, Renewable Energy, Vol. 28 (2003) No. 28, p. 1013-1027.

[19] S.D. Kwon: Uncertainty analysis of wind energy potential assessment. Applied Energy, Vol. 87(2010) No. 3, p. 856-865.

[20] H. Aksoy, Z.F. Toprak, A. Aytek, N.E. Ünal: Stochastic generation of hourly mean wind speed data, Renewable Energy, Vol. 29 (2004) No. 14, p. 2111-2131.

[21] S. Mathew, G.S. Philip, C. M. Lim: Analysis of wind regimes and performance of wind turbines, advances in wind energy conversion technology, New York: Springer (2011). 